\title{
High-level expression of sugar inducible gene2 (HS/2) is a negative regulator of drought stress tolerance in Arabidopsis
}

\author{
Nirmala Sharma, Yarnel Bender, Kerry Boyle and Pierre R Fobert ${ }^{*}$
}

\begin{abstract}
Background: HIGH-LEVEL EXPRESSION OF SUGAR INDUCIBLE GENE2 (HSI2), also known as VAL1, is a B3 domain transcriptional repressor that acts redundantly with its closest relative, HSI2-LIKE1 (HSL 1), to suppress the seed maturation program following germination. Mutant hsi2 hs/1 seedlings are arrested early in development and differentially express a number of abiotic stress-related genes. To test the potential requirement for HSI2 during abiotic stress, hsi2 single mutants and plants overexpressing HSI2 were subjected to simulated drought stress by withholding watering, and characterized through physiological, metabolic and gene expression studies.

Results: The hsi2 mutants demonstrated reduced wilting and maintained higher relative water content than wild-type after withholding watering, while the overexpressing lines displayed the opposite phenotype. The hsi2 mutant displayed lower constitutive and ABA-induced stomatal conductance than wild-type and accumulated lower levels of ABA metabolites and several osmolytes and osmoprotectants following water withdrawal. Microarray comparisons between wild-type and the hsi2 mutant revealed that steady-state levels of numerous stress-induced genes were up-regulated in the mutant in the absence of stress but down-regulated at visible wilting. Plants with altered levels of HSI2 responded to exogenous application of ABA and a long-lived ABA analog, but the hsi2 mutant did not show altered expression of several ABA-responsive or ABA signalling genes $4 \mathrm{hr}$ after application.

Conclusions: These results implicate $\mathrm{HSI} 2$ as a negative regulator of drought stress response in Arabidopsis, acting, at least in part, by regulating transpirational water loss. Metabolic and global transcript profiling comparisons of the hsi2 mutant and wild-type plants do not support a model whereby the greater drought tolerance observed in the hsi2 mutant is conferred by the accumulation of known osmolytes and osmoprotectants. Instead, data are consistent with mutants experiencing a relatively milder dehydration stress following water withdrawal.
\end{abstract}

Keywords: Abiotic stress, Abscisic acid, Gene expression, Microarray, Transcriptome, Water stress

\section{Background}

Water deficit is a major environmental factor limiting plant fitness and productivity [1]. With patterns of global climate change likely to increase the severity of drought stresses in the future [2], the development of crop plants better adapted to water-limited environments is a priority for sustainable agriculture.

Plants have evolved unique mechanisms to monitor water availability in their environment and adapt to it $[3,4]$. In response to mild water deficits, low cellular water

\footnotetext{
* Correspondence: Pierre.Fobert@nrc-cnrc.gc.ca

National Research Council Canada, 110 Gymnasium Place, Saskatoon, SK S7N ow9, Canada
}

potential can be avoided by balancing water uptake and loss through stomatal closure, increased cuticle thickness and enhanced root growth. Should these adaptations be insufficient to restore physiological water potential within the cell, more dramatic measures may be deployed. These are generally distinguished as dehydration avoidance, dehydration tolerance and drought escape [3], although all three responses are highly integrated [5]. A key aspect of dehydration avoidance is the accumulation of compatible solutes (osmolytes), including sugars, sugar alcohols, amino acids and organic acids, that increase cellular osmotic potential, prevent water loss and maintain turgor [6]. Dehydration tolerance consists of measures to protect cell constituents from damage and entails the production 
of antioxidants and chaperones, such as dehydrins and late-embryogenesis abundant (LEA) proteins [7].

The plant hormone abscisic acid (ABA) plays a pivotal role in coordinating multiple aspects of adaptation to drought stress [8]. Stomatal closure, the accumulation of osmolytes and the synthesis of protective proteins are all correlated with drought-induced increases in endogenous ABA levels. Mutants defective in ABA synthesis or perception are more susceptible to drought, while treatment with exogenous ABA enhances drought tolerance and induces the expression of numerous dehydration-stress responsive genes and proteins. However, several dehydration stress-induced genes do not respond to exogenous application of ABA in Arabidopsis thaliana (L.) $[9,10]$, suggesting the existence of both ABA-dependent and -independent signal transduction pathways.

Physiological and biochemical aspects of drought adaptation are underpinned by extensive transcriptional re-programming [9]. Drought-induced transcriptomes from several plant species have been reported (reviewed in [11]) and reveal profound changes in numerous processes, including growth, amino acid and carbohydrate metabolism, photosynthesis, protection against oxidative stress, phosphorylation, membrane transport, secretion, cell wall expansion, and hormone homeostasis [10,12-15]. Functional characterization efforts have identified the role of a number of drought-induced transcripts, including many transcription factors, in driving adaptive responses [10,16-18]. Differential gene expression in response to abiotic stress is likely associated with changes in chromatin conformation, such as those mediated by histone tail modifications and chromatin remodeling complexes $[19,20]$. Identification and characterization of the factors and mechanisms involved is only now being elucidated and an active area of research.

One group of proteins that may be implicated in regulating plant chromatin conformation is the HIGHLEVEL EXPRESSION OF SUGAR-INDUCIBLE GENE2 (HSI2) clade of B3 domain proteins. The B3 domain is a plant-specific basic DNA-binding domain originally identified as the third and $\mathrm{C}$-terminus proximal basic domain of the transcription factor ABSICIC ACID INSENSITIVE3 (ABI3) (reviewed in [21]). There are three members of the HSI2 clade in Arabidopsis; HSI2, also known as the VIVIPAROUS ABI3-LIKE1 (VAL1), HSI2LIKE1 (HSL1, also known as VAL2) and HSI2-LIKE2 (HSL2, also known as VAL3) [22-24]. Additional features shared by HSI2 clade proteins include the CW domain and EAR motif. HSI2 and HSL1 also contain a putative plant homeodomain (PHD)-like zinc finger domain absent from HSL2. The CW and PHD-like domains are associated with chromatin remodelling factors, while the EAR motif is a transcriptional repression domain. Functional analysis of HSI2 has demonstrated that the protein is a potent EAR-dependent transcriptional repressor [23] and can repress ABI3-mediated transactivation from the $\mathrm{Sph} / \mathrm{RY}$ element, the cognate DNA binding site of B3 domain factors [25]. Loss-of-function mutations in HSI2 result in the expression of seed storage proteins in vegetative tissues $[23,24]$. The deregulation of embryonic and seed maturation programs in vegetative tissues is more dramatic in hsi2 hsl1 (val1 val2) double mutants, which produce callus and ectopic embryo-like structures when seedlings are cultured in the presence of sucrose. Among genes highly expressed in the double mutant seedlings are ABI3, FUSCA3 (FUS3) and LEAFY COTYLEDON2 (LEC2), which encode key regulators of seed maturation and constitute the AFL (ABI3/FUS3/ LEC2) clade of B3 domain proteins. It has been proposed that HSI2 clade proteins suppress the seed maturation program following germination by repressing the transcription of AFL clade genes and/or competing with the encoded transcription factors for $\mathrm{Sph} / \mathrm{RY}$ sites in the promoter of target genes [22,24,25].

The recent analysis of the hsi2-4 allele, which creates a cysteine to tyrosine substitution in the PHD domain, revealed a potential role for this HSI2 domain in the repression of a subset of seed-specific genes during seedling development and in the deposition of the repressive chromatin mark H3K27m3 on target genes [26]. Interestingly, $h s i 2-4 h s l$ double mutants were morphologically normal, indicating a unique function for the HSI2 PHD domain.

Molecular genetic analysis of the HSI2 clade has focused on hsi2 hsll double mutants at the seedling stage. The potential role of these factors at other times in the plant life cycle remains largely unexplored, despite the fact that HSI2 and HSL1 are expressed in many tissues [23]. A cursory analysis of gene ontology (GO) annotation terms enriched in the list of genes differentially expressed in hsi2 and hsi2 hsl1 mutants [24,26] identified response to abiotic stimulus as highly significant (data not shown). This prompted us to test whether HSI2 might regulate drought tolerance during the vegetative stage of the plant life cycle. Through loss- and gain-of-function analyses, HSI2 is shown to act as a negative regulator of drought tolerance in Arabidopsis, possibly through a mechanism involving reduced transpirational water loss.

\section{Results}

To investigate the role of HSI2 in modulating drought responses, the levels and/or integrity of the gene were stably altered in transgenic Arabidopsis plants. For loss-of-function analysis, two T-DNA insertion alleles were identified (Figure 1A). Both mutant alleles show 


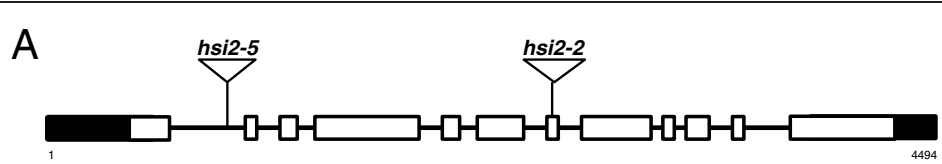

B

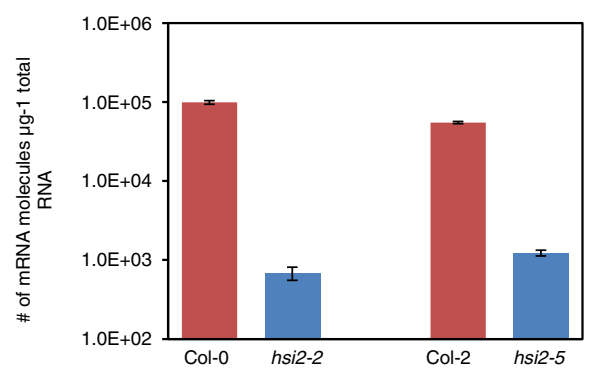

C

HSI2 (Genomic)

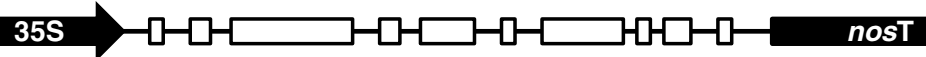

D

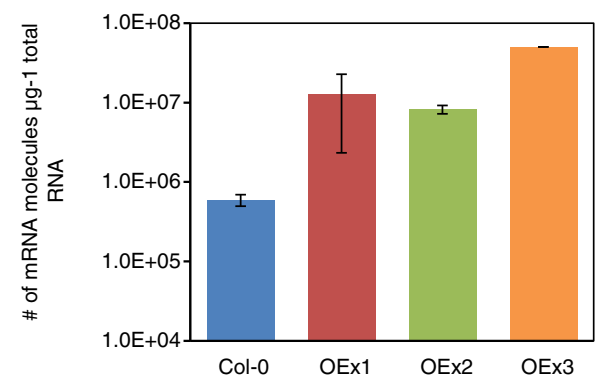

E

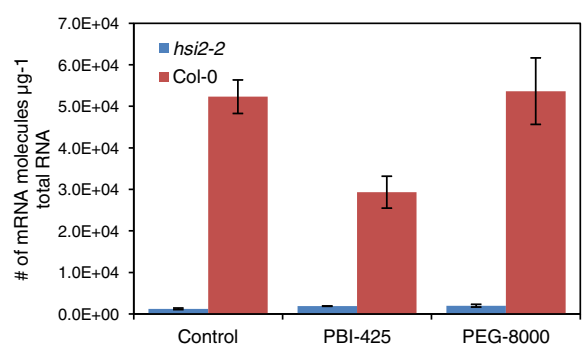

$\mathrm{F}$

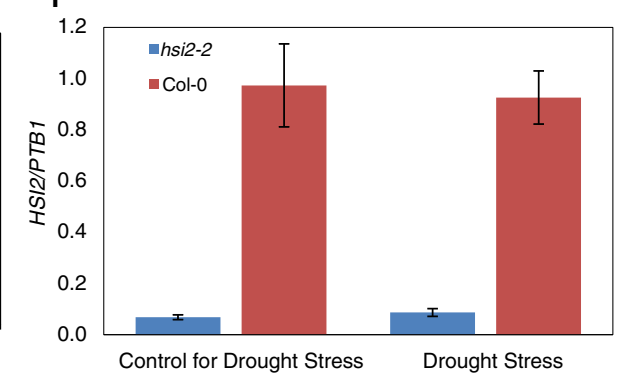

Figure 1 HSI2 insertional mutants and overexpressing lines. (A) Diagram of the structure of the HS/2 gene (At2g30470) showing the position of T-DNA insertions (triangles). Open boxes indicate exons, while black boxes indicate untranslated regions. The transcriptional start site is indicated by the number 1. With the exception of T-DNA sizes, the diagram is drawn to scale. (B) Kinetic RT-PCR analysis of HSI2 mRNA abundance in the mutants and corresponding wild-types (hsi2-2/Col-0 and hsi2-5/Col-2). (C) Diagram of the structure of the HS/2 transgene. Open boxes indicate exons; 35S, Cauliflower mosaic virus $35 \mathrm{~S}$ promoter; nosT, terminator region from the Agrobacterium tumefaciens nopaline synthase gene. (D) Kinetic RT-PCR analysis of HSI2 mRNA abundance in transgenic plants and untransformed Col-0. (E) Kinetic RT-PCR analysis of HSI2 mRNA abundance in 14 day old seedlings of hsi2-2 and Col-0, 4 h following treatment with 25 HM PBI425 or 20\% PEG 8000. (F) Kinetic RT-PCR analysis of HSI2 mRNA abundance in 3-week-old leaves of hsi2-2 and Col-0 grown in soil saturated to field capacity or following watering withdrawal and development of visible wilting symptoms. For (F), amplification of the housekeeping gene encoding polypyrimidine tract-binding protein1 (PTB1, AT3G01150) was used as control to normalize expression data. All values represent the averages of three biological replicates, each analyzed three times (technical replicates) \pm standard error.

minimal expression of HSI2 when compared to corresponding wild-types, Col-0 for hsi2-2 and Col-2 for hsi2-5 (Figure 1B). For gain-of-function analysis, the HSI2 coding region was placed under the control of the Cauliflower mosaic virus $35 \mathrm{~S}$ promoter (35S:HSI2) and used to generate transgenic plants in Col-0 (Figure 1C). Three independent lines showing increased levels of HSI2 transcripts (Figure 1D, OEx lines) were further studied. 


\section{Disruption of $H S / 2$ by T-DNA insertions confers better drought tolerance}

The response of hsi2 mutants to drought was examined by subjecting plants to a simulated drought stress regime in environmentally controlled growth chambers. To minimize sources of variation attributed to the amount, and the initial moisture content, of the soil in pots, wild-type and corresponding mutant plants were grown side-by-side in the same containers. Both hsi2 mutants displayed reduced rates of wilting after withholding water, by as much as $9 \%$, compared to their wild-type counterparts (Figure 2A). The hsi2 mutants also maintained higher cellular water levels, by as much as $30 \%$, measured as leaf relative water content (RWC; Figure 2B). Additional experiments using different pot sizes and potting mixes yielded similar findings (data not shown). These results indicate that hsi2 mutants have increased tolerance to drought, due at least in part to a reduced rate of water loss from leaves.

\section{HSI2 overexpression enhances drought sensitivity}

The three independent OEx lines evaluated showed an early onset and greater severity of visual wilting, and poorer recovery from a drought stress compared to untransformed controls (Figure 2C-E). Differences in visible wilting were most pronounced at 6 and 7 days after withholding water, with OEx lines displaying between 48-60\% and $35-45 \%$ higher wilting rates, respectively (Figure 2C). When plants were re-watered at the end of the drought period and allowed to recover for $24 \mathrm{~h}, 66 \%$ of the wildtype plants recovered compared to 16,24 , and $44 \%$ in the OEx lines (Figure 2D, 2E). Together with data obtained from loss-of-function mutants, the results implicate HSI2 as a negative regulator of drought tolerance.

\section{$H S / 2$ altered plants respond to an exogenously applied ABA}

To study the possible interaction between HSI2 and ABA during drought stress, we assessed the drought response of the hsi2-2 mutant and line OEx1 pre-treated with

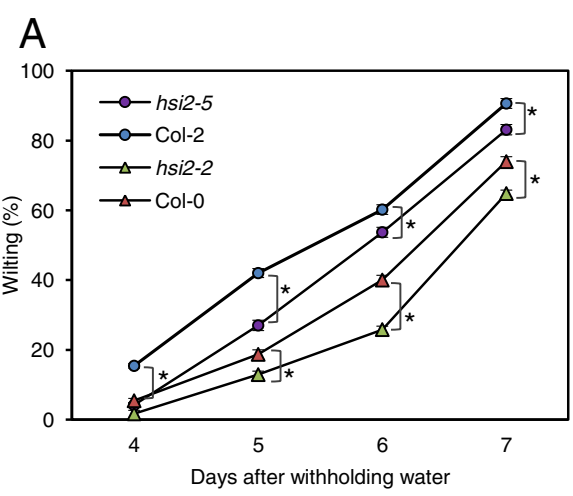

C

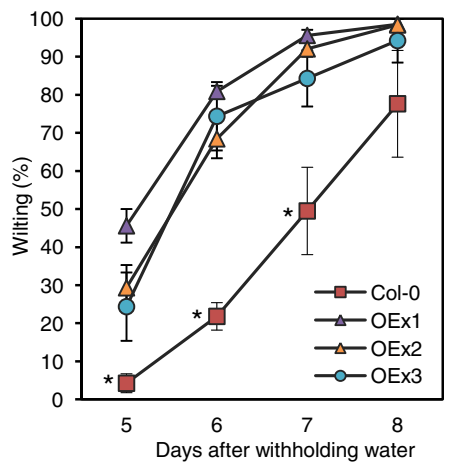

B

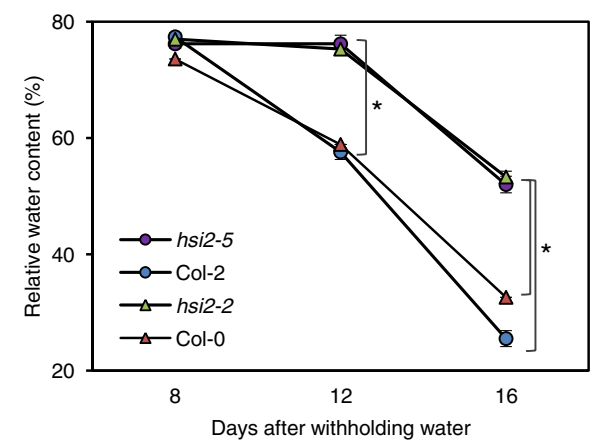

$\mathrm{E}$

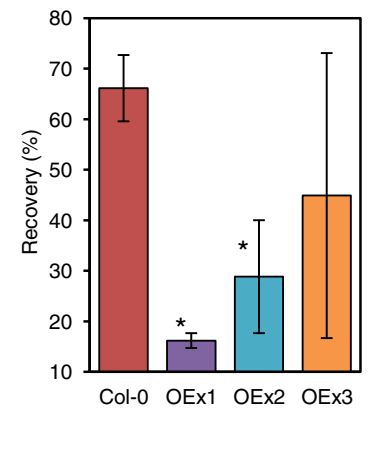

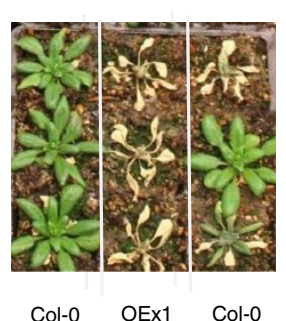

Col-0 OEx1 Col-0

Figure 2 Response of $h s i 2$ mutants and 35S:HSI2 overexpressing lines to simulated drought stress. (A) Wilting rates of hsi2 mutants and corresponding wild-types. (B) Leaf relative water content of the hsi2 mutants and corresponding wild-types. (C) Rate of wilting of three independent 35S:HSI2 lines and untransformed wild-type (Col-0). (D) Recovery from drought $24 \mathrm{~h}$ upon re-watering. (E) A representative photograph showing the recovery phenotype of Col-0 and 35S:HSI2 line OEx1. For (A) and (B), the hsi2-2 mutant compares with Col-0 and hsi2-5 compares with Col-2; each wild-type and mutant combination were planted in the same sets of containers. Statistical significance, indicated by an asterisk $\left(^{*}\right)$, was determined by a paired Student's $t$-test $(p \leq 0.05)$. Data are presented as means of three replicates containing 12 plants each \pm standard error of means. In some cases where the standard errors are very small, the graph symbols may obscure the bars. Experiments were repeated at least three times with similar results. 
(+)-ABA and (+)-8'-acetylene ABA, also known as PBI425 [27]. This synthetic analog is resistant to 8 'hydroxylation, the principal ABA catabolic pathway in Arabidopsis $[12,28]$. Consequently, PBI425 is more persistent in planta and has stronger hormonal activity $[29,30]$. The ability of PBI425 to enhance drought tolerance in Arabidopsis has been shown to be mediated through ABA signalling [29]. Whether PBI425 and ABA act through the same signaling pathway can be tested by binding to the family of PYL receptors. Nevertheless, available information indicate that PBI425 offers several advantages over natural ABA when the sustained presence of hormonal activity is required, as is the case in this study.

In control treatments where plants were root-drenched with water prior to water withdrawal, hsi2-2 plants displayed less wilting, and OEx1 plants more wilting, than the wild-type at all observation times (Figure 3A and 3B). When plants were given a root-drench treatment with ABA $(30 \mu \mathrm{M})$ or PBI425 $(20 \mu \mathrm{M})$ before withholding water, the onset of visible wilting was delayed in all genotypes by three to four days (until $7-10$ days after treatment), after which the mutant continued to display less wilting, and OEx1 more wilting, than the wild-type at all observation times (Figure 3A and 3B). Recovery from wilting upon re-watering was evaluated under control and ABA analog treatments, where the hsi2-2 mutant recovered the best upon re-watering, followed by the wild-type and OEx1 plants (Figure 3C). However, the overall recovery upon re-watering was lower in all genotypes pretreated with PBI425.

\section{Hsi2-2 mutants display lower stomatal conductance}

Lower stomatal conductance has been associated with higher drought tolerance in many plant species including Arabidopsis [31,32]. Accordingly, stomatal conductance was measured to investigate possible mechanisms underpinning the increased drought tolerance in the hsi 2 mutant. Leaf stomatal conductance in well-watered hsi2-2 plants was $77 \%$ of wild-type values (Figure 4A), suggesting lower constitutive stomatal conductance. Pretreatment with PBI425 resulted in a 2.4-fold reduction in stomatal conductance in the wild-type and a 4.4-fold reduction in hsi2-2 (Figure 4B), with the hsi2-2 mutant displaying $42 \%$ of wild-type stomatal conductance following pre-treatment with PBI425 (Figure 4B). These results suggest that the mutant is able to better maintain water status through regulation of stomatal properties and that loss of HSI2 does not substantially affect associated $\mathrm{ABA}$ signaling.

\section{The $h$ si2-2 mutant accumulates less ABA metabolites during drought stress}

To examine the involvement of HSI2 in ABA metabolism during the drought response, levels of endogenous $\mathrm{ABA}$ and its metabolites were measured in leaves of $h s i 2-2$, OEx1, and Col-0 before drought stress, at visible wilting, and upon rehydration. In the absence of drought, levels of all metabolites were similar in the three genotypes (Figure 5). Endogenous ABA content increased dramatically under drought stress in all three genotypes, and reverted to near pre-stress levels upon re-watering. However, hsi2-2 accumulated relatively lower levels of ABA during drought and was better able to recover to original levels after re-watering compared to OEx1 or Col-0. Levels of ABA metabolites, namely ABA-glucose ester (ABA-GE), dihydrophaseic acid (DPA), phaseic acid (PA), and transABA ( $t$-ABA) also increased substantially under drought stress in all genotypes (Figure 5). The hsi2-2 mutant accumulated less of these metabolites than Col-0, while OEx1 contained levels comparable to Col-0, with the exception of $t$-ABA, which was lower in 35S:HSI2. Consistent with the reports that the 8'-hydroxylation pathway is the most important ABA catabolic pathway in Arabidopsis [12,28], the products of this pathway (PA and DPA), were the most abundant during drought stress and after rehydration (Figure 5). Unlike ABA, the levels of most metabolites remained much higher after rehydration than they were pre-drought, with DPA levels remaining as high as during drought stress. These results indicate that drought-induced ABA biosynthesis and catabolism is of smaller amplitude in the mutant line, resulting in a more modest increase in both $\mathrm{ABA}$ and $\mathrm{ABA}$ metabolites, while rehydration reversed the effect of drought on $A B A$ biosynthesis but not ABA catabolism.

\section{The hsi2-2 mutant accumulates lower levels of stress- induced metabolites during drought stress}

To ascertain the role of HSI2 during stress-induced metabolic reprogramming, levels of several compounds were measured by undirected metabolic profiling and compared between hsi2-2 and Col-0 before drought stress, at visible wilting, and upon rehydration. Overall, drought treatment increased levels of numerous metabolites previously reported to be drought-inducible [33], including soluble sugars, sugar alcohols, amino acids, organic acids and antioxidants (Figure 6; Additional file 1). Levels of several metabolites were also depleted under drought conditions. With some exceptions, most metabolites reverted closer to non-stress levels upon rehydration.

Relatively few of the stress-regulated metabolites were found to accumulate differentially in the two genotypes tested (Figure 6; Additional file 1). These included sugars (fructose and glucose), amino acids (proline, tryptophan and aspartate), and the sugar alcohol ribitol, levels of which were lower in the mutant than the wild-type under drought stress. Similarly, the products of the lysine degradation pathway, N6-acetyllysine, 2-aminoadipate and pipecolate, which have been shown to accumulate under 


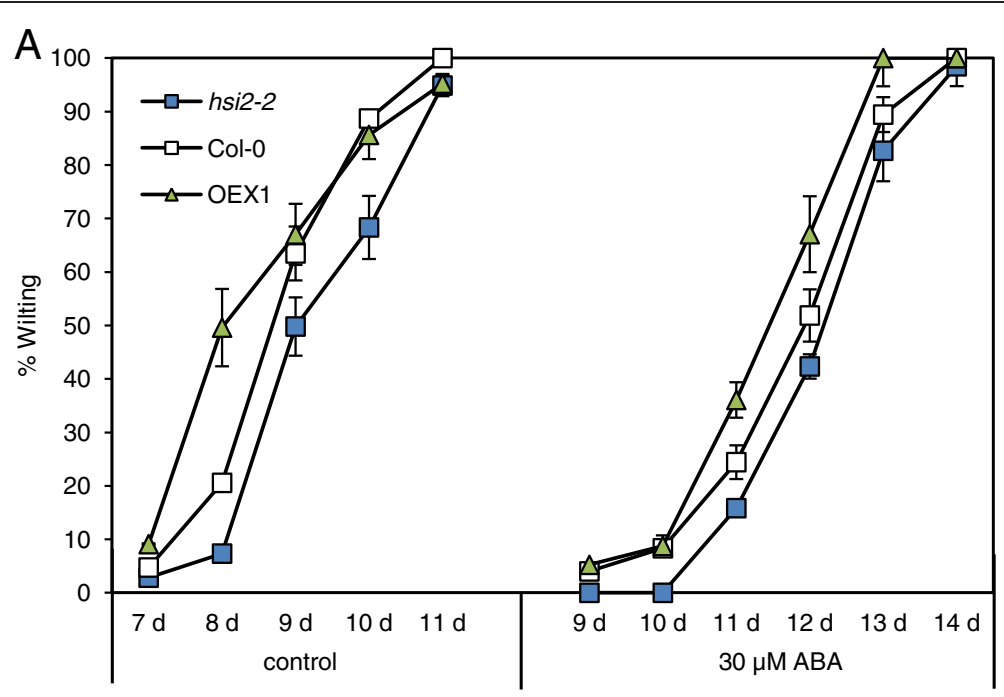

B

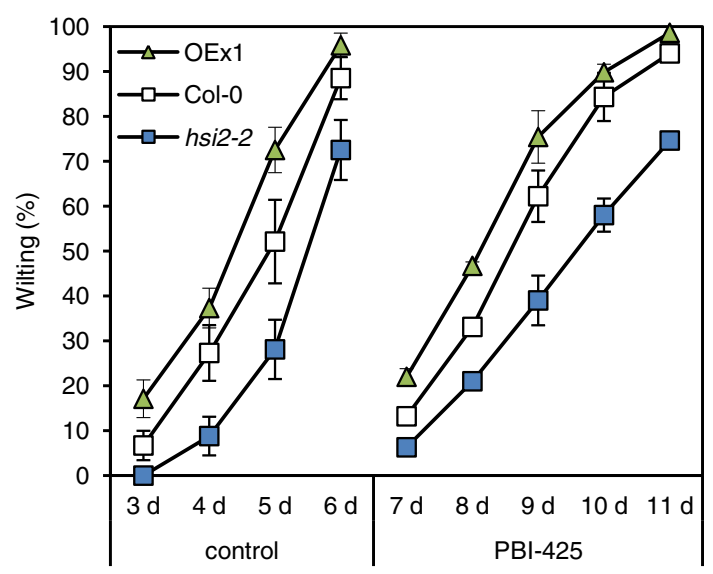

C

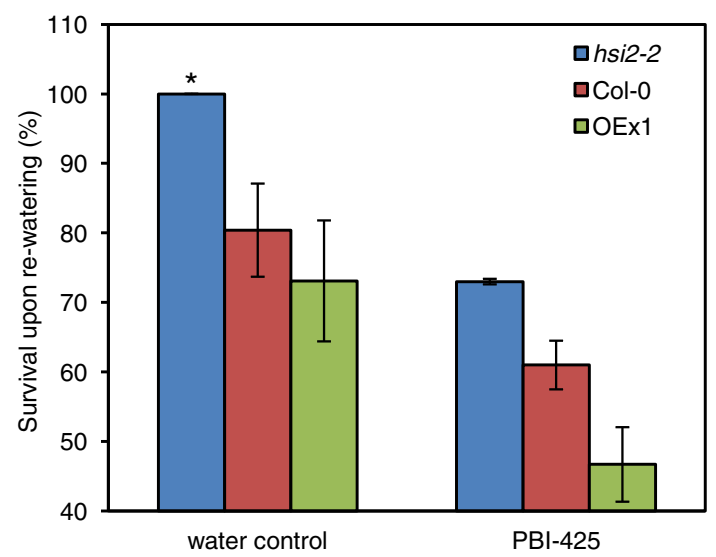

Figure 3 Response of $h s i 2$ mutants and 35S:HSI2 overexpressing lines to simulated drought stress. (A-B) Rate of wilting of wild-type (Col-0), mutant (hsi2-2), and 35S:HSI2 line (OEx1) treated with (+)-ABA (A) or the ABA analog PBI425 (B). (C) Recovery from drought upon re-watering (at $24 \mathrm{~h}$ after re-watering under control and PBI425 treatments). Three-week-old plants were root drenched with $0.05 \%$ ethanol as control treatment (left side), $30 \mu \mathrm{M}$ ABA or $20 \mu \mathrm{M}$ PBI425, dissolved in $0.05 \%$ ethanol (right side) to field capacity and watering was stopped thereafter. Data are presented as means of three replicates of 18 plants each \pm standard error of means. In some cases where the standard errors are very small, the graph symbols may obscure the bars. These experiments were repeated at least twice with similar results. 
osmotic stress in Brassica napus [34] and suggested to have osmoprotectant properties in bacteria (pipecolate [35]) were also lower in the mutant compared to wild-type under drought conditions. In contrast, hsi2-2 had significantly higher levels of ferulate, an intermediate of the phenylpropanoid pathway, before and during drought (Figure 6). Lower levels of known stress-induced osmolytes and osmoprotectants in the hsi2 mutant at wilting are consistent with plants experiencing relatively milder dehydration stress than the wild-type.

\section{Analysis of drought transcriptomes indicates that drought-induced genes are down-regulated in the hsi2 mutant}

To characterize the consequences of loss of HSI2 function on gene expression relevant to drought stress, genomewide expression analysis was undertaken with the hsi2-2 mutant under a normal watering regime (no stress; stage 0 ) and after withholding watering, before the onset of visible wilting (stage 1) and at visible wilting (stage 2). Gene expression in the mutant was compared with its wild-type (Col-0) under each watering regime by co-hybridization using dual color microarrays. Genes corresponding to 441 unique AGIs were identified as being differentially expressed ( $p \leq 0.05,1.5$-fold cut-off) at stage 0 , and the numbers of differentially expressed unique AGIs increased with the severity of drought to 785 (stage 1) and 1400 (stage 2) (Additional files 2 and 3). Consistent with differences in drought responses observed between the mutant and wildtype (Figures 2, 3, 4), the most significant GO enriched terms for biological process identified in lists of differentially expressed genes were response to stress and response to biotic or abiotic stimulus (Table 1). The relative $p$-values for these terms decreased with the severity of the drought treatment. The identity of genes differentially regulated under the three watering regimes changed considerably. Of the 441 genes differentially expressed at stage 0,335 (76\%) are specific to this stage and only 48 genes were differentially regulated under all watering regimes. For representative genes, microarray results were validated by kinetic reverse-transcriptase PCR (k-RT-PCR) (Figure 7).

Genes differentially expressed in the $h s i 2$ mutant were compared with those regulated in response to drought stress. Given the poor overlap of genes reported as being differentially expressed during drought stress in different studies (see $[11,12,36]$ ), genes regulated by drought in our datasets were identified by comparing probe intensity values from individual channels between stage 2 and stage 0 in Col-0. The direction of change for these genes in our datasets was overwhelmingly the same as those from published studies employing whole genome microarrays and similar drought regimes (Additional file 3).

Genes found to be induced in response to drought stress in our dataset ( $>2.5$-fold) were predominantly down-

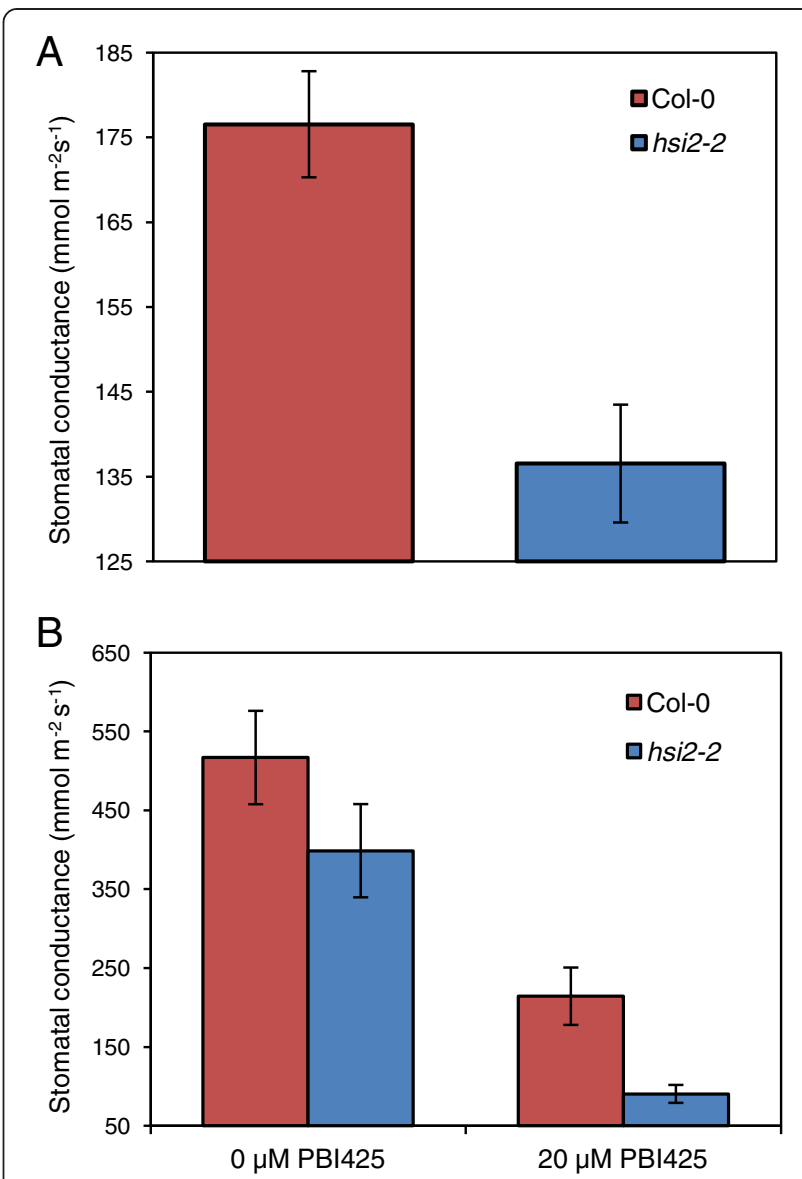

Figure 4 Stomatal conductance in the hsi2-2 mutant and corresponding wild-type (Col-0). Stomatal conductance was measured in fully developed young leaves (A) $1 \mathrm{~d}$ after watering or (B) $72 \mathrm{~h}$ after root drench treatment with 0 or $20 \mu \mathrm{M}$ PBI425 (in $0.05 \%$ ethanol). Data presented as means of three replicates \pm standard error of means, each containing 4-6 plants and measurements from two leaves per plant. The experiments were repeated twice with similar results.

regulated when differentially expressed in the hsi 2 mutant (vs. Col-0) at stage 2 (>92\%), while genes repressed by drought were mostly up-regulated (93\%) when differentially expressed in the $h s i 2$ mutant at this drought stage (Additional file 3). Similar patterns were observed when genes differentially expressed in $h s i 2$ vs. Col-0 at stage 2 were compared to published lists of drought-regulated transcripts of Huang et al. [12] or the progressive drought $(\mathrm{pDr})$ and ABA-induced genes of Harb et al. [14] (Additional file 3). Analysis of GO terms enriched in genes induced by drought and down-regulated in $h s i 2$ vs. Col- 0 at stage 2 identified predominantly droughtassociated processes, including response to abiotic stimulus, ABA, and water deprivation (Table 2). These terms were not enriched in the gene list induced by drought and up-regulated in hsi2. 


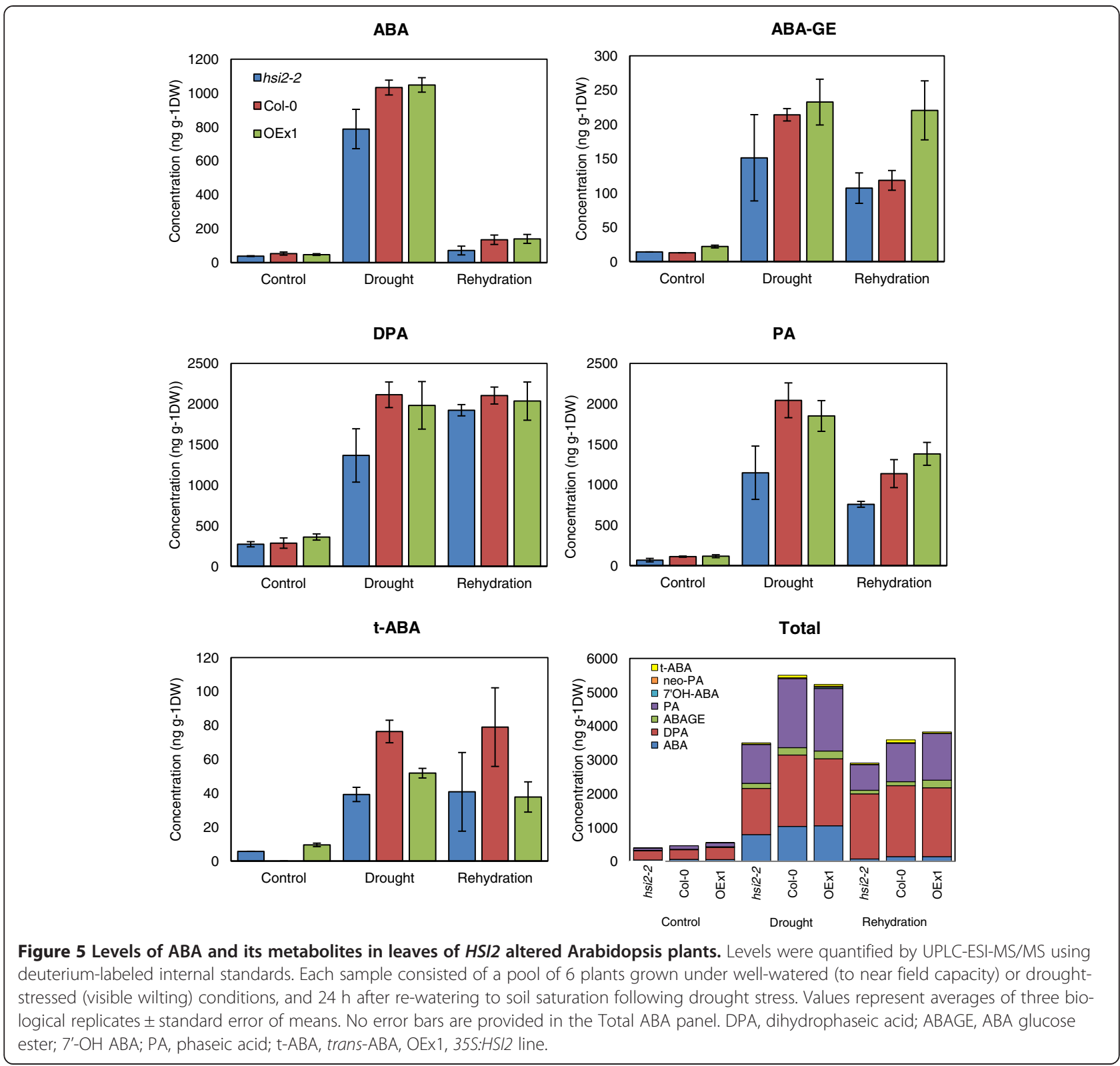

Comparisons of gene lists differentially expressed in hsi 2 vs. Col-0 at stage 2 to lists from Wilkins et al. [36] or the moderate drought $(\mathrm{mDr})$ treatments of Harb et al. [14] failed to identify a clear trend between the direction of change in $h s i 2$ vs. Col-0 relative to drought inducibility/repression (Additional file 3). However, drought-induced genes from these moderate drought treatments, the pDr of Harb et al. [14], and the present study were predominantly up-regulated when differentially expressed in $h s i 2$ vs. Col- 0 at stage 1 , reaching over $80 \%$ for genes induced in mDr1 and mDr10 of Harb et al. [14] and up-regulated in hsi2 vs. Col-0 at stage 1
(Additional file 3). Genes repressed by drought or ABA did not show the complimentary trend and no clear trends were identified in the stage 0 data. Notable GO terms enriched in drought-regulated genes differentially expressed in $h s i 2$ vs. Col-0 at stage 0 or stage 1 include response to abiotic, temperature and chemical stimuli, and oxidoreductase activity (Table 2). Taken together, these results indicate that the hsi2 mutant constitutively expresses a number of drought- and ABA-responsive genes but under wilting conditions, the induction or repression of drought- and ABA-regulated genes is attenuated relative to the wild-type. 
Accumulation of $A B A-$-responsive transcripts in response to the ABA analog PBI425 is not compromised in the hsi2 mutant

To ascertain the possible involvement of HSI2 during the early responses to ABA and osmotic stress, RNA was extracted from two-week-old seedlings of Col-0 and hsi2-2, $4 \mathrm{~h}$ after treatment with PBI425 or PEG 8000, and the expression of selected genes was analyzed by k-RT-PCR.
Steady-state mRNA levels of five well-known ABA responsive marker genes (COR78/RD29A, RD29B, ERD6, KIN1, and RD22 [14,15]) continued to show wild-type induction to PBI425 and PEG 8000 in the hsi2-2 mutant, while $R A B 18$ mRNA levels were higher in hsi2 than Col-0 following exposure to the ABA analog (Figure 8).

Of 6 genes tested that have been implicated in ABA signaling, mRNA levels for three $(A B F 2, A B I 2$ and $H A B 1)$
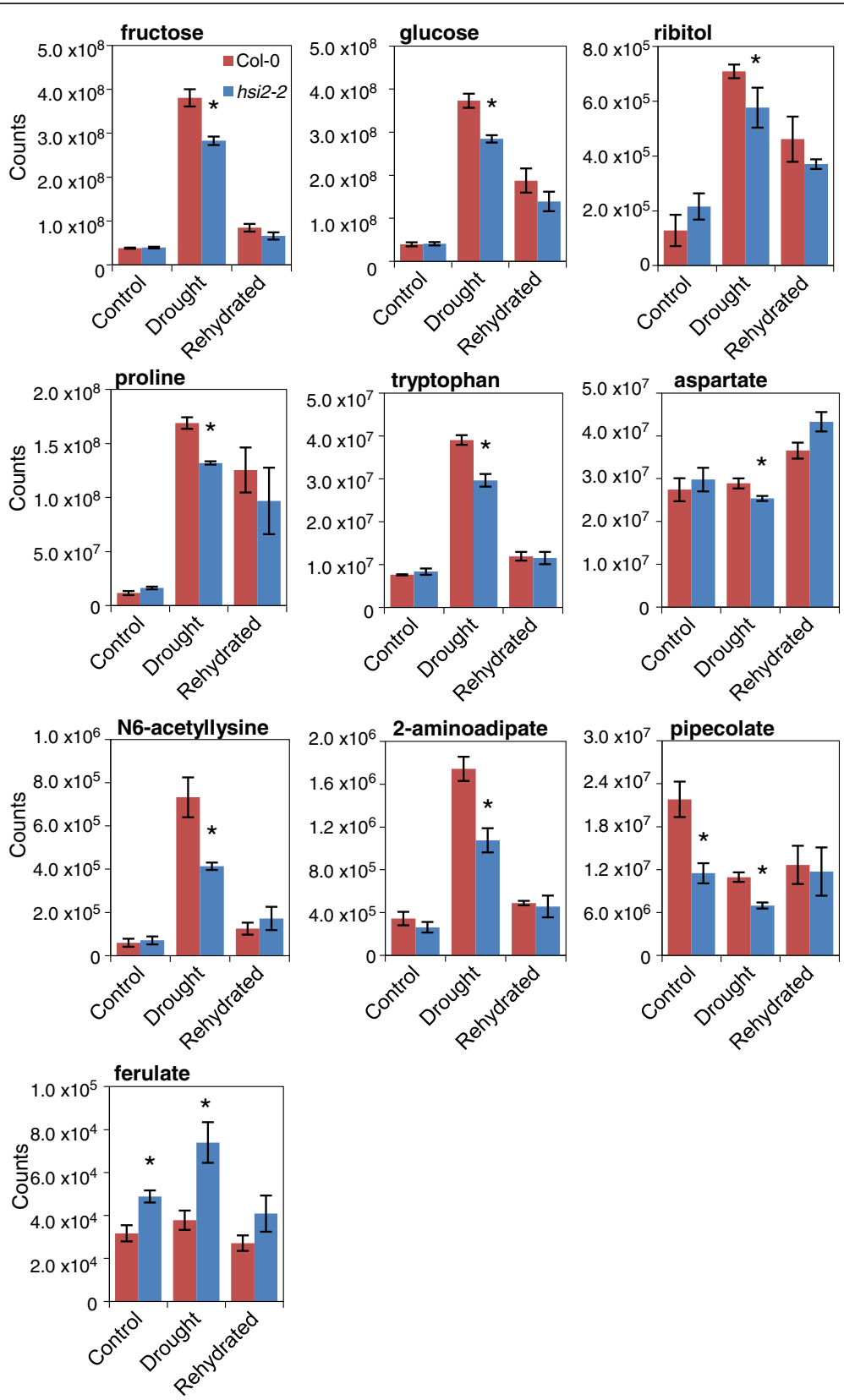

Figure 6 Drought-related levels of selected metabolites showing significant changes in leaves of the hsi2-2 mutant. Each sample consisted of a minimum of 6 plants grown under well-watered (to near field capacity) or drought-stressed (visible wilting) conditions, and $24 \mathrm{~h}$ after re-watering to soil saturation following drought stress. Values represent averages of three biological replicates \pm standard error. Treatments statistically different by ANOVA ( $p \leq 0.05)$ are marked by asterisks. 
Table 1 Gene Ontology classification terms enriched in the hsi2-2 mutant compared to Col-0 under different simulated drought regimes

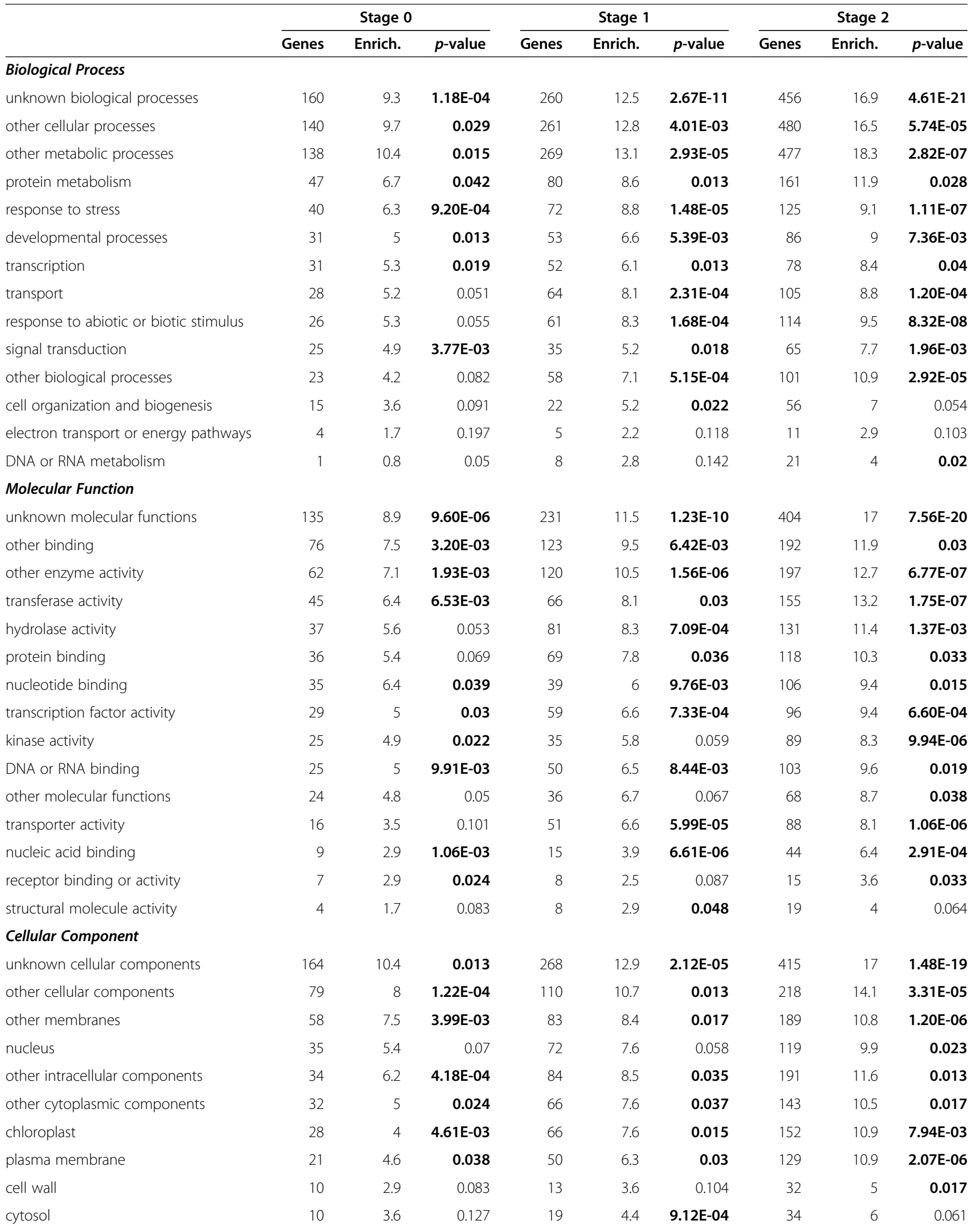


Table 1 Gene Ontology classification terms enriched in the hsi2-2 mutant compared to Col-0 under different simulated drought regimes (Continued)

\begin{tabular}{lllrrrrrrr}
\hline plastid & 7 & 2.7 & $\mathbf{0 . 0 1 2}$ & 12 & 3.3 & 0.086 & 45 & 6.9 & 0.061 \\
mitochondria & 6 & 2.6 & $\mathbf{4 . 6 8 E - 0 3}$ & 19 & 4.3 & 0.112 & 46 & 6.1 & 0.06 \\
extracellular & 6 & 2.5 & 0.163 & 12 & 3.5 & $\mathbf{0 . 0 2 7}$ & 34 & 5.2 & $\mathbf{3 . 2 1 E}-\mathbf{0 4}$ \\
ER & 4 & 1.9 & 0.177 & 10 & 2.5 & 0.057 & 15 & 3.8 & 0.102 \\
ribosome & 2 & 1.1 & $\mathbf{0 . 0 3 5}$ & 7 & 3.4 & 0.119 & 18 & 3.9 & 0.084 \\
Golgi apparatus & 1 & 0.7 & 0.142 & 4 & 1.7 & 0.159 & 11 & 3.2 & 0.114 \\
\hline Enrichments were performed at http://bar.utoronto.ca/ntools/cgi-bin/ntools_classification_superviewer.cgi. Statistically significant values $(p<0.05)$ are in bold.
\end{tabular}

Stage 0 , watered to field capacity; Stage 1, dry soil, no visible wilting; Stage 2, visible wilting.

[37] were highly induced by PBI425 but displayed identical expression patterns in Col-0 and hsi2-2 (Figure 8). Transcripts for PHOSPHOLIPASE D 1 (PLD $\alpha 1$ ) and ANACO19, both positive regulators of ABA signalling [38,39], showed higher levels of expression in the mutant than wild-type following treatment with PBI425. With PLD 1 , mRNA levels in hsi2 were also higher in the control and PEG 8000 treatments, suggesting constitutive differences in expression rather than altered responses to ABA and drought. Expression of ERA1, which encodes a farnesyl transferase and a negative regulator of ABA signalling [40], was not inducible by PBI425 and showed no consistent differences between Col-0 and $h s i 2-2$.
Two genes implicated in mediating drought stress (CBF1 and RD20) were highly inducible by PBI 425 (Figure 8). While hsi2-2 and Col-0 displayed identical patterns of expression for RD20 in control and PBI 425 treatments, expression of the transcription factor $C B F 1$ was higher in hsi2-2 following treatment with the ABA analog. Expression of neither MYB15, MYB88 nor ERECTA were inducible by PBI425; however, levels of MYB15 in hsi2-2 were lower in the control, but not the PBI425 treatment and MYB88 levels were higher in hsi2 following PBI425 treatment. Levels of ERECTA mRNA also showed a trend of being higher in the hsi2 mutant under different treatments, although differences were

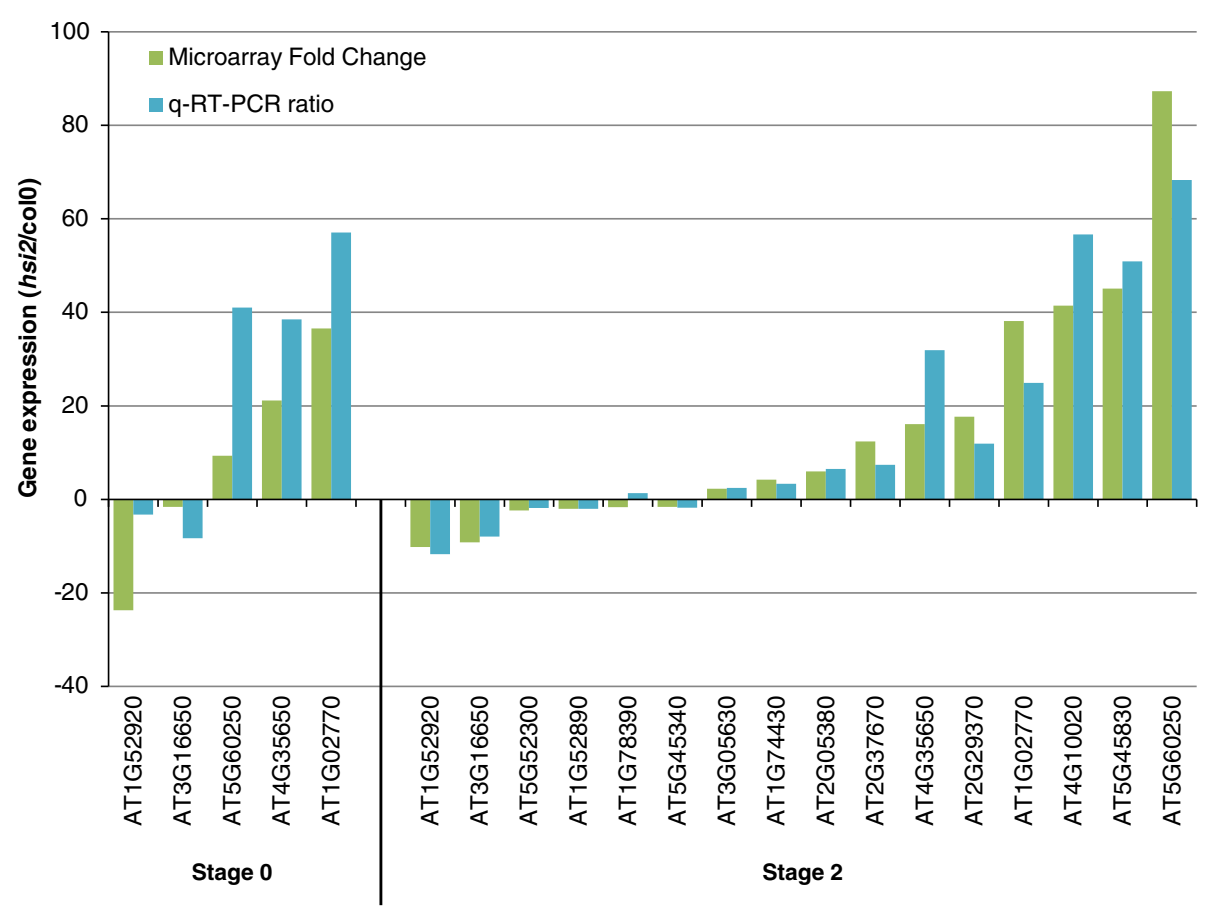

Figure 7 Kinetic RT-PCR validation of microarray analyses for a representative subset of genes. CDNA was synthesized from the areal portion of well watered plants (Stage 0) and at visible drought (Stage 2). Amplification of the housekeeping gene encoding polypyrimidine tract-binding protein1 (PTBpa, AT3G01150) was used as control to normalize PCR expression data. All values represent the averages of three biological replicates, each analyzed three times (technical replicates). Values from microarray expression are ratios of averaged, normalized fluorescent signals. 
Table 2 Representative GO terms enriched in the $h s i 2-2$ mutant versus Col-0 and regulated by drought stress

\begin{tabular}{|c|c|c|c|c|c|}
\hline Description & GO term & Enrichment & $p$-value & Example & AGI \\
\hline \multicolumn{6}{|c|}{ Induced by Drought and Down-regulated at Stage 2 (hsi2:Col-0) } \\
\hline$P^{*}$ response to abiotic stimulus & GO:0009628 & 2.03 & $5.27 \mathrm{E}-04$ & ABA receptor GCR2 & At1g52920 \\
\hline$P$ response to temperature stimulus & GO:0009266 & 3.51 & $3.41 \mathrm{E}-04$ & RAB18, RD29B & At5g66400; At5g52300 \\
\hline$P$ response to light intensity & GO:0009642 & 8.51 & 5.27E-04 & ZAT12 & At5g59820 \\
\hline$P$ response to water deprivation & GO:0009414 & 4.28 & $2.98 \mathrm{E}-03$ & RAB18, ANAC019 & At5g66400; At1g52890 \\
\hline$P$ response to abscisic acid stimulus & GO:0009737 & 3.51 & $1.18 \mathrm{E}-03$ & RAB18, RD29B & At5g66400; At5g52300 \\
\hline$F^{*}$ oxygen binding & GO:0019825 & 3.63 & 4.93E-04 & CYP705A12, $-15,-19$ & At5g42580; At3g20080; At3g20100 \\
\hline \multicolumn{6}{|c|}{ Induced by Drought and Up-regulated at Stage 2 (hsi2:Col-0) } \\
\hline P secondary metabolism & GO:0019748 & 5.48 & $6.12 \mathrm{E}-03$ & 4-coumarate-CoA ligase 2 & At3g21240 \\
\hline C* plasma membrane & GO:0005886 & 4.98 & $8.54 \mathrm{E}-03$ & LTP2 & At2g38530 \\
\hline \multicolumn{6}{|c|}{ Repressed by Drought and Up-regulated at Stage 2 (hsi2:Col-0) } \\
\hline P steroid biosynthesis & GO:0006694 & 12.34 & 7.97E-03 & DWARF 4 & At3g50660 \\
\hline P carbohydrate metabolism & GO:0005975 & 2.22 & 4.50E-04 & Glycosyl hydrolase 9C2, 9B8, and 17 & At1g64390; At2g32990; At2g05790 \\
\hline P secondary metabolism & GO:0019748 & 2.3 & $9.31 \mathrm{E}-03$ & ESM1, CAD4 & At3g14210; At3g19450 \\
\hline P carboxylic acid metabolism & GO:0019752 & 2.28 & 1.10E-03 & LOX2 & At3g45140 \\
\hline F water transporter activity & GO:0005372 & 9.73 & $1.52 \mathrm{E}-04$ & DELTA-TIP, PIP1 & At3g16240; At3g61430 \\
\hline F carbon-oxygen lyase activity & GO:0016835 & 4.31 & $1.27 \mathrm{E}-03$ & $a-$ and $\beta$-carbonic anhydrase 1 & At3g52720; At3g01500 \\
\hline C endomembrane system & GO:0012505 & 1.53 & $1.55 \mathrm{E}-05$ & RKL1, LTP7 & At1g48480; At2g15050 \\
\hline C plastid thylakoid & GO:0031976 & 3.18 & $1.57 \mathrm{E}-04$ & $\beta$-carbonic anhydrase 1 & At3g01500 \\
\hline C anchored to membrane & GO:0031225 & 3.65 & $1.31 \mathrm{E}-04$ & TOO MANY MOUTHS & At1g80080 \\
\hline \multicolumn{6}{|c|}{ Repressed by drought and Down-regulated at Stage 2 (hsi2:Col-0) } \\
\hline P DNA metabolism & GO:0006259 & 6.94 & $7.51 \mathrm{E}-04$ & KRYPTONITE & At5g13960 \\
\hline F protein binding & GO:0005515 & 2.64 & $6.11 \mathrm{E}-03$ & Variant in methylation 4 & At1g66040 \\
\hline \multicolumn{6}{|c|}{ Induced by Drought and Down-regulated at Stage 1 (hsi2:Col-0) } \\
\hline F electrochemical potential-driven transporter activity & GO:0015290 & 5.88 & 1.65E-03 & GPT2, ZIFL2 & At1g61800; At3g43790 \\
\hline \multicolumn{6}{|c|}{ Induced by Drought and Up-regulated at Stage 1 (hsi2:Col-0) } \\
\hline P nitrogen compound biosynthesis & GO:0044271 & 5.07 & $8.23 \mathrm{E}-03$ & GLN1.3 & At3g17820 \\
\hline$P$ response to chemical stimulus & GO:0042221 & 2.23 & 7.92E-03 & FIBRILLIN & At4g04020 \\
\hline F oxidoreductase activity & GO:0016491 & 2.54 & $8.39 \mathrm{E}-04$ & FERREDOXIN3 & At2g27510 \\
\hline F protein ser/thr phosphatase activity & GO:0004722 & 5.43 & 6.47E-03 & PP2CA2 & At1g30220 \\
\hline \multicolumn{6}{|c|}{ Repressed by Drought and Up-regulated at Stage 1 (hsi2:Col-0) } \\
\hline P cellular carbohydrate metabolism & GO:0044262 & 4.24 & 3.01E-03 & Cellulose synthase-like B1 & At2g32610 \\
\hline F hydrolase activity & GO:0016787 & 1.86 & $9.05 \mathrm{E}-03$ & Methyl IAA esterase & At5g58310 \\
\hline
\end{tabular}

P carbohydrate metabolism

GO:0005975

GO:0019748

GO:0016835

GO:0012505

O:0031976

(hsi2:Col-0)

Repressed by drought and Down-regulated
9.05E-03 
Table 2 Representative GO terms enriched in the $\boldsymbol{h s i 2 - 2}$ mutant versus Col-0 and regulated by drought stress (Continued)

Induced by Drought and Up-regulated at Stage 0 (hsi2:Col-0)

$P$ phenylpropanoid metabolism GO:0009698

$P$ response to temperature stimulus GO:0009266

$\begin{array}{lll}19.64 & 5.15 \mathrm{E}-05 & \text { TT6G, CAD } \\ 7.17 & 8.46 \mathrm{E}-03 & \text { COR15B } \\ 3.09 & 3.72 \mathrm{E}-03 & \text { COR15B, AGL19 } \\ 3.56 & 3.15 \mathrm{E}-03 & \text { TTG6, CAD }\end{array}$

At3g51240; At1g09500

$P$ response to abiotic stimulus

GO:0009628

$3.15 \mathrm{E}-03$

TTG6, CAD

At2g42530

F oxidoreductase activity

GO:001649

Repressed by Drought and Down-regulated at Stage 0 (hsi2:Col-0)

$P$ protein modification GO:0006464

F kinase activity

GO:0016301

6.34

7.37E-04

HDAC 18

At2g42530; At4g22950

At3g51240; At1g09500

* P, Biological Process; F, Biological Function; C, Cellular Component.

In cases where multiple related classifications were significant, only a subset is listed. Lists with fewer than 3 genes are not shown. Enrichments were performed at http://bioinformatics.psb.ugent.be/ATCOECIS using $p<0.05$. 

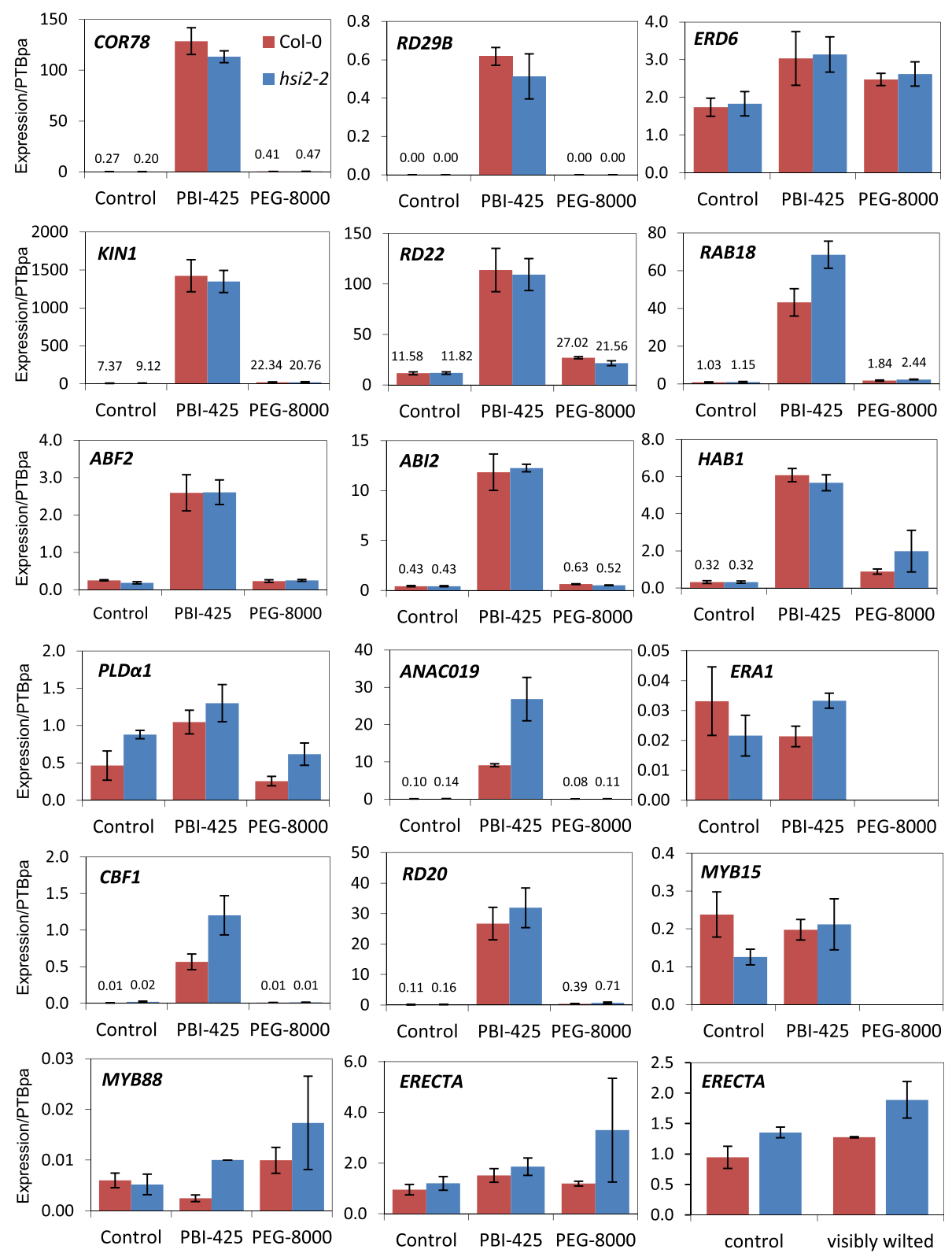

Figure 8 Kinetic RT-PCR analysis of gene expression. CDNA was synthesized from total RNA from 14-day old seedlings treated with $25 \mu \mathrm{M}$ PBI425 or 20\% PEG 8000 for $4 \mathrm{~h}$. Amplification of the housekeeping gene encoding polypyrimidine tract-binding protein1 (PTBPa, AT3G01150) was used as control to normalize expression data. All values represent the averages of three biological replicates, each analyzed three times (technical replicates) \pm standard error. In cases where expression levels were too small to graph, values are indicated in writing. If no bar is visible and no value indicated, the analysis was not performed.

more obvious in the PEG 8000 treatment. Differences in ERECTA expression were also observed in rosette leaves before and after drought stress (Figure 8). Thus, the loss of HSI2 function did not affect the expression of most genes tested within $4 \mathrm{~h}$ of PBI 425 treatment. A longerterm study may reveal latent changes in ABA-inducible expression, if any, of these genes in the mutant compared to the wild type.

\section{Discussion}

Our analyses establish a role for HSI2 in response to water stress during the vegetative stage of the Arabidopsis life cycle. Specifically, loss of HSI2 function results in plants better able to maintain physiological water potential under limiting water conditions, as indicated by reduced wilting and higher leaf RWC (Figure 2), while overexpression of $H S I 2$ yields a complementary phenotype. These results are 
consistent with HSI2 acting as a negative regulator. The ability of the hsi2 mutant to avoid low water potential is likely attributed, at least in part, to lower stomatal conductance (Figure 4), effectively reducing transpirational water loss. Consistent with this phenotype, the mutant constitutively expresses higher levels of genes implicated in reducing water loss or improving water use. These include ERECTA, a positive regulator of water use efficiency through control of stomatal density and conductance [31] and PHOSPHOLIPASE D $\alpha 1$, a positive regulator of ABAinduced stomatal movement and early-stage drought resistance [38] (Figure 8). Conversely, the transcription factor GTL1, a negative regulator of water use efficiency through its effect on stomatal density [32] is downregulated in $h s i 2$ at stage 0 . Despite these changes in gene expression, no differences in stomatal density were obvious between the hsi2 mutants and their corresponding wild-types in preliminary analyses. Stomatal density in Arabidopsis is developmentally controlled and affected by the environment [41]. Accordingly, more detailed analyses are required of both stomatal density and aperture. It is noteworthy that HSI2 transcripts are likely preferentially expressed in guard cells in the absence of stress [42,43], and thus could be involved in the direct or indirect regulation of guard cell genes.

An unanticipated finding of this study was the reduced levels of numerous metabolites and gene transcripts associated with tolerance to dehydration stress measured in the hsi2-2 mutant, compared to Col-0. This includes lower levels of ABA and several ABA metabolites (Figure 5) as well as osmolytes and osmoprotectants (Figure 6). Global analysis of gene expression revealed that most genes up-regulated under severe drought conditions are expressed at lower levels in hsi2-2 than in Col-0 (Additional file 3). Among GO terms highly enriched in these lists of genes include response to abiotic stimulus, response to water deprivation, and response to $\mathrm{ABA}$ stimulus (Table 2). It is noteworthy that levels of many of these metabolites continue to be induced, and levels of gene transcripts up-regulated, under drought conditions; it is the magnitude of change that is dampened in the mutant compared to the wild-type. These results are consistent with hsi2-2 plants experiencing, or at least perceiving, a milder dehydration stress than the wild-type under the conditions tested. One possible explanation for these findings is that the ability of $h s i 2-2$ seedlings to maintain RWC and avoid low water potential for a longer time than Col-0 delays the onset of cellular dehydration and attenuates its severity. Indeed, based on our metabolite and gene expression data, there is no evidence to indicate that hsi2-2 seedlings should have greater tolerance to dehydration stress. This includes expression analysis of several genes associated with dehydration stress in seedlings treated with a PEG solution that simulates dehydration stress (Figure 8). The possibility that HSI2 affects drought tolerance by regulating other, and possibly, novel pathways cannot be excluded at this time and requires further investigation. Among the GO terms most significantly enriched in genes differentially expressed in $h s i 2$ vs Col- 0 are endomembranes and chloroplasts and represent potential targets for future studies. Included in the endomembranes group are a number of transporters (amino acid, water channel, polyamine transporters, and membrane) such as MATE efflux family proteins, proton-dependent oligopeptide transport (POT) family proteins, auxin efflux carriers, $A B C$ transporters, a delta TIP (water channel protein), sugar and nitrate transporters and a cytochrome oxidase. Recently, differential expression of genes encoding pumps and transporters were associated with increased drought tolerance of rice near isogenic lines [13].

Seeds acquire desiccation tolerance during the final stages of maturation. Desiccation tolerance is also an important component of drought tolerance, and parallels between the two processes have been drawn previously [7]. HSI2 is known to be required for repression of seed maturation genes upon germination and the transition to vegetative growth $[22,24,26]$. In the absence of direct evidence for altered HSI2 activity in response of drought stress (Figure 1 and [23]), it could be argued that increased drought tolerance of the hsi2 mutant is a secondary effect triggered by the derepression of seed desiccation genes during vegetative growth. Indeed, transcripts of four genes encoding LEA proteins are constitutively up-regulated in leaves of the hsi2 mutant (Additional file 2) and GO terms consistent with antioxidant activity (e.g. flavonoids, oxidoreductases) are enriched (Table 2). However, these changes are unlikely to account for the altered dehydration avoidance responses described above. Overall, 23\% of genes differentially regulated in $h s i 2$ at stage 0 are also differentially expressed during the later stages of seed maturation, with $16 \%$ showing the same directionality of change in both conditions (data not shown). These values are lower than those observed in seedlings of hsi2 mutants [24,26]. Given that HSI2 regulation of seed maturation genes is dependent on sugar [22], the differences are likely attributed to the inclusion of sucrose in the media used to grow seedlings. An alternative possibility is that HSI2 may only be required for a limited period of time during vegetative development for repressing seed maturation programs, as reported for PICKLE [44]. Of note, the seedling studies reported up-regulation of gene encoding master regulators of seed maturation (LEC1 and AFL clade B3 transcription factors). Of these, only transcripts of FUS3 were detected above background in leaves three-week-old Col-0 or hsi 2 plants, and its expression was not altered by loss of HSI2. Thus, activation of seed maturation genes in leaves of the hsi2 mutant is likely to involve 
different signalling events. In a number of organisms, genes regulating embryogenic events have been shown to be required at later stages of development, including in response to stress $[45,46]$. Thus, a role for HSI2 in repressing seed maturation genes upon germination and later, in response to water stress, is not unprecedented.

Our results indicate that HSI2 is not absolutely required for ABA signalling during drought stress. Both the (+)-ABA and its analog PBI425, which increase drought tolerance by activating ABA responses and ABA signalling $[12,29]$, continued to effectively induce greater drought tolerance in both the hsi2 mutant and 35S:HSI2 plants without altering the relative tolerance of these genotypes compared to the wild-type (Figure 3). PBI425 was also effective at reducing stomatal conductance in the mutant, with levels continuing to be lower than the wild-type following treatment (Figure 4). Furthermore, the expression of several genes involved in ABA synthesis and signalling was unaltered in the mutant $4 \mathrm{hr}$ after treatment with PBI425 (Figure 8), suggesting the mutant is not affected in its ability to respond to this hyperactive ABA analog, and presumably to $\mathrm{ABA}$ itself. In cases where gene expression was different between the mutant and wild-type following PBI425, it was typically also altered in the absence of PBI425, suggesting an ABA-independent cause. Although differences in the levels of several ABA-responsive genes were observed between $h s i 2$ and wild-type when plants displayed visible wilting symptoms, these were measured several days following water withdrawal, thus complicating interpretation. Altered patterns of gene expression under these conditions are likely attributed, at least in part, to different levels of ABA in the mutant at wilting, which in turn we hypothesize to result for better maintenance of water potential in the mutant. Although the effects of PBI425 are mediated through ABA signalling, global transcript profiling indicates that the two compounds do not act in an identical fashion [29]. Furthermore, exogenous application of growth regulators is limited in its ability to resolve the function of endogenous ones. For these reasons, additional research will be required to study the relationship between HSI2 and ABA. This should include evaluating the role of endogenous ABA and ABA signalling by epistatic analysis of $h s i 2$ and mutants defective in ABA synthesis or perception, such as nced3.

Given that HSI2 is an EAR-dependent transcriptional repressor [23], it could act to limit the transcription of a subset of drought-inducible genes when water is abundant. Such target genes would be constitutively derepressed upon loss of HSI2 function, as observed in comparisons of $h s i 2$ and Col-0 transcriptomes at stage 0 (Table 2 and Additional file 3), conferring a benefit to the mutant upon initial exposure to limiting water conditions. Several previously reported stress responsive genes from Arabidopsis, including SFR6, LEA4-5, ATPAL1, AtCIMS,
EGY3, ATCP1, ATHAL3, ATHSP-70, FAR1, and GDH1, are differentially expressed in hsi2 in the absence of a drought stress. Notably, nearly all of the differentially expressed genes involved in cellular/transmembrane transport (of sugar, lipids and metal ions) are up-regulated in the mutant suggesting more active transport and detoxification than in the wild-type. Also consistent with detoxification, possibly of reactive oxygen species known to accumulate in response to abiotic stress, is the enrichment of GO terms for oxidoreductases and flavonoids in hsi2 at stage 0 (Table 2). We speculate that HSI2mediated transcriptional repression is naturally relieved upon perception of limiting water conditions. Transcript levels of HSI2 do not appear to be altered in response to stress (Figure 1 and [23]), suggesting that de-repression of drought-related HSI2 targets is likely mediated by translational or post-translational modification of HSI2. Several EAR-containing plant proteins have been shown to be post-translationally modified by phosphorylation or poly-ubiquitination, providing a potential means of relieving their repressive effects on gene expression (reviewed in [47]).

\section{Conclusions}

By subjecting single $h s i 2$ mutants and plants overexpressing HSI2 to simulated drought stress by withholding watering, we have demonstrated a role for the putative chromatin remodelling factor HSI2 during drought stress at vegetative stage of the Arabidopsis life cycle life. Although elucidating the exact role of HSI2 will require additional research, available information indicates that it fulfils a negative role in maintaining physiological water potential under limiting water conditions and as such represents a potential target for genetic manipulation towards the development of crops better suited for cultivation under water-limited environments. Formal demonstration that HSI2 activity is regulated in response to drought stress will be important to resolving its biological role at this stage of the plant life cycle. The identification of direct targets for HSI2, the dynamics of HSI2 binding to these targets and the associated epigenetic state of the targets following water withdrawal will be required to clarify the involvement and mechanism of action of HSI2 in mediating drought-related gene expression.

\section{Methods}

\section{Plant materials and growth conditions}

Arabidopsis thaliana (L.) Heynh. was used throughout. T-DNA insertion lines, hsi2-2 (SALK_088606) and hsi2-5 (WiscDsLox388F10) were identified from the Salk Institute Genomic Laboratory Genomic database (http://signal. salk.edu; [48] and obtained from the Arabidopsis Biological Resource Centre (ABRC, http://abrc.osu.edu/). The locations of T-DNA insertions were confirmed by 
sequencing of PCR fragments and plants homozygous for the T-DNA insertions were identified by PCR (oligos used for PCR amplification are listed in Additional file 4).

To generate plants overexpressing HSI2, the genomic protein-coding region of At2g30470 was amplified by PCR from the BAC T6B20 (ABRC) and inserted sequentially into pDONRZeo (Invitrogen, http://www.invitrogen.com) and the pK7WG2 [49] derivative pER330 (E. Risseeuw and R. Datla, unpublished) using Gateway technology (Invitrogen). The integrity of the resulting 35S:HSI2 gene was confirmed by sequencing. Transgenic plants were generated by dipping inflorescences of Col-0 [50] in a suspension of Agrobacterium tumefaciens GV3101 (MP90) harboring the modified T-DNA binary plasmid and subjecting the resulting seeds to antibiotic selection $\left(30 \mathrm{mg} \mathrm{l}^{-1}\right.$ kanamycin). Independent $T_{3}$ or $T_{4}$ lines expressing the transgene were analyzed.

Unless otherwise noted, seeds of different genotypes were stratified for 2 days at $4^{\circ} \mathrm{C}$, sown on Sunshine \#4 potting mix (Sun Gro Horticulture, http://www.sungro. $\mathrm{com} /$ ) and transferred to environment-controlled growth chambers with a $16-\mathrm{h}$ photoperiod $\left(200 \mu \mathrm{mol} \mathrm{m}{ }^{-2} \mathrm{~s}^{-1}\right)$ at $22 / 20^{\circ} \mathrm{C}$ (day/night). All samples for gene, hormone and metabolite analysis were collected at the same time of day to minimize circadian effects, immediately frozen in liquid nitrogen and stored at $-80^{\circ} \mathrm{C}$.

\section{Drought treatments and measurements}

Plants were watered as needed to maintain the soil moisture near field capacity and fertilized weekly with $20 \mathrm{~N}: 20 \mathrm{P}: 20 \mathrm{~K}$ until three-week-old, at which point they were subjected to drought stress by withholding water. Visual wilting, indicated by progressive loss of lush green color of leaves and drooping of leaf blades and petiole, was monitored daily thereafter until more than $80 \%$ plants were wilting. At the end of the observation period, plants were re-watered to field capacity, and recovery was recorded $24 \mathrm{~h}$ thereafter. Drought response (visual wilting) and recovery from wilting upon rewatering were evaluated in three independent batches of plants, originating from at least 2 different seed lots, with 36-72 plants observed per batch. Leaf water status was measured when more than $50 \%$ of the plants started to wilt. Results are presented as leaf Relative Water Content $(\mathrm{RWC}=$ fresh weight-dry weight/turgid weight-dry weight) and as percentage of wilted or dead plants at, or over, a certain period of time. In experiments evaluating the effect of the ABA analog on the onset and progress of drought stress, three-week-old plants were subjected to root-dip treatment with $30 \mu \mathrm{M}$ of $(+)$-ABA or $20 \mu \mathrm{M}$ of the long-lived synthetic ABA analog, (+)-8'-acetylene ABA (PBI425) in $0.05 \%$ ethanol v/v [27] and watering was withheld thereafter. The concentrations of $A B A$ and the PBI425 were chosen based on information from the published literature [12]. Preliminary dose-response experiments established these levels as being effective at delaying visual wilting in Col-0 without undesired side effects, such as the accumulation of anthocyanins and leaf curling, that were observed when higher concentrations of PBI425 or (+)-ABA were applied. Because the hsi 2 mutant responded very similarly to $A B A$ and PBI425, and PBI425 offers several advantages over natural $\mathrm{ABA}$ when the sustained presence of hormonal activity is required, the synthetic analog was used in subsequent experiments. Student's $t$-test $(\mathrm{p} \leq 0.05)$ was performed to identify significant differences between treatments.

\section{Measurement of stomatal conductance}

Stomatal conductance was measured from fully expanded young leaves from well-watered or PBI425treated plants using a steady state leaf porometer (Model SC-1,Decagon Devices, http://www.decagon.com) following the manufacturer's instructions. Porometer readings were taken from the adaxial side (lower surface) of leaves by placing the sensor head at the widest part of the leaf and holding it in place until the measurement was complete $(\sim 30$ seconds/reading $)$. Measurements were taken from 2-4 leaves per plant and 8-12 different plants from each genotype and treatment. Porometer readings taken different leaves of a plant were averaged to derive a single value, and genotype and treatment average were calculated thereafter. Measurements were performed between 9:30 am to 11:30 am, two $\mathrm{h}$ after lights were turned on in the cabinets.

\section{Quantification of ABA and metabolites by LC-MS/MS}

Analysis was conducted at the National Research Council, Saskatoon, by UPLC-ESI-MS/MS (http://www. nrc-cnrc.gc.ca/eng/solutions/advisory/plant_hormone.html) as described in Chiwocha et al. [51]. For ABA profiling, tissues for analysis were harvested from the aerial portion of 3-week-old, well-watered (soil saturated to field capacity), drought-stressed (showing wilting) and rewatered (to field capacity, $24 \mathrm{~h}$ ) plants. Each sample consisted of material pooled from six plants.

\section{Metabolomic profiling and analysis}

For metabolic profiling analyses, tissues were harvested from the aerial portion of 3-week-old, well-watered (to soil saturation, near field capacity), drought-stressed (showing visible wilting) and re-watered (to soil saturation for $24 \mathrm{~h}$ ) plants and frozen immediately in liquid nitrogen and stored at $-80^{\circ} \mathrm{C}$ until analyzed. Samples were collected in three biological replicates per genotype and each sample (65-250 mg) consisted of material pooled from six plants. Frozen tissues were freeze-dried and analyzed by Metabolon Inc (http://www.metabolon.com) as 
described by Oliver et al. [52]. Briefly, samples were extracted and prepared for analysis using Metabolon's standard solvent extraction method. The extracted samples were split into equal parts for analysis on the GC/MS and LC/MS/MS platforms. Also included were several technical replicate samples created from a homogeneous pool containing a small amount of all samples considered in the study. A total 156 named biochemicals were identified. Following log transformation and imputation with minimum observed values for each compound, ANOVA contrasts were used to identify biochemicals that differed significantly between two genotypes at various time points.

\section{Microarray analysis}

Gene-expression profiles were generated using Arabidopsis $4 \times 44 \mathrm{~K}$ oligonucleotide microarrays (http:// www.agilent.com). Total RNA was isolated using the Qiagen RNAeasy Plant Mini Kit (http://www.qiagen. com) and quality was assessed using an Agilent-2100 Bioanalyzer. Each sample co-hybridized consisted of rosette leaves from four plants. Four independent biological replicates were analyzed at stage 0 (well-watered to field capacity) and three replicates at each of stage 1 (dry soil following withholding watering but before visible wilting) and stage 2 (visible wilting). Labeling and hybridization were performed following the manufacturer's protocol using Agilent's QuickAmp Labeling kit for two-color microarrays and incorporated a dye swap design. After washing, arrays were scanned and signals converted to expression data using GenePix 4000B scanner (GenePix Pro. 6.1, http://www.moleculardevices. com). Further in-house analysis was performed in GeneSpring GX.10 (Agilent). Additional analyses of differentially expressed genes were conducted using the following web-based tools; the Classification SuperViewer (The BAR; http://bar.utoronto.ca/ntools/cgi-bin/ ntools_classification_superviewer.cgi), ATCOECIS (http:// bioinformatics.psb.ugent.be/ATCOECIS), and Athena (http:// www.bioinformatics2.wsu.edu/cgi-bin/Athena/cgi/analysis_select.pl). To compare expression levels of the wild-type before and after drought, single channel intensity values were obtained using the formula intensity calculator plugin in BASE 2.14.0 (http://base.thep.lu.se) and normalized with the Limma software package for R [53], using robust spline for intraarray and quantile for interarray normalizations. MIAME complaint data have been submitted to GEO (Accession \# GSE39347).

\section{Kinetic polymerase chain reaction analysis}

Total RNA was isolated using the Agilent Plant RNA Isolation Mini Kit and treated with amplification grade DNase I (Invitrogen). The first-strand cDNA was synthesized using SuperScript ${ }^{\bullet}$ II reverse transcriptase (Invitrogen) and kinetic Polymerase Chain Reaction was performed on an MX3005P spectrofluorometric thermal cycler (Stratagene, http://www.stratagene.com) as described by Sharma et al. [54]. Amplification of the housekeeping gene encoding polypyrimidine tract-binding protein1 (PTBpa; AT3G01150) was used as control to normalize expression data. The list of genes analyzed and primers used in k-RTPCR experiments are presented in Additional file 5.

For analysis of gene expression in response to PBI425 and polyethylene glycol, seeds were surface sterilized, stratified as above, and germinated on agar solidified 0.5× Murashige and Skoog (MS) medium (Sigma, http:// www.sigma.com) in $150 \times 15 \mathrm{~mm}$ Petri plates in a controlled environment chamber $\left(80-100 \mu \mathrm{mol} \mathrm{m}^{-2} \mathrm{~s}^{-1}\right.$ light Intensity, $16-\mathrm{h}$ photoperiod, $24^{\circ} \mathrm{C}$ ). Fourteen-day-old plants were flooded with PBI425 $(25 \mu \mathrm{M})$ or $20 \%$ PEG 8000 on the germination plates and incubated at room temperature for $4 \mathrm{~h}$ prior to tissue harvest.

\section{Additional files}

Additional file 1: Heat maps of metabolic responses in Col-0 and hsi2-2 to drought stress.

Additional file 2: Annotated list of probe sets differentially regulated in leaves of $h s i 2-2$ at each stages of drought.

Additional file 3: Summary of comparisons between genes found to be regulated by drought in the present study and the literature.

Additional file 4: Summary of comparisons between genes found to be regulated by drought in the present study and the literature, considering directions of expression changes. Genes with $>2.5 \times$ difference in average signal intensity between Col-0 at stage 0 vs stage 2 were compared. Duplicate AGI codes were removed.

Additional file 5: List of genes and primer sequences used in this study.

\section{Abbreviations}

35S: Cauliflower mosaic virus 355 promoter; ABA: Abscisic acid; ABA-GE: ABAglucose ester; ABI3: Absicic acid insensitive3; AFL: ABI3/FUS3/LEC2;

DPA: Dihydrophaseic acid; FUS3: FUSCA3; GO: Gene ontology; HSI2: High-level expression of sugar inducible gene2; HSL1: HSI2-Like1; HSL2: HSI2-Like2; k-RT-

PCR: Kinetic reverse-transcriptase PCR; LEA: Late-embryogenesis abundant; LEC2: Leafy cotyledon2; mDr: Moderate drought; OEx: Over expression (lines show increased levels of HSI2 transcripts); PA: Phaseic acid; pDr: Progressive drought; PHD: Plant homeodomain; PLDa1: Phospholipase Da1; RWC: Relative water content; t-ABA: Trans-ABA; VAL1: Viviparous ABI3-Like1.

\section{Competing interests}

Patent applications have been filed on the use of HSI2 to enhance drought tolerance (International Patent Application PCT/CA2010/000754, United States Patent Application 13320813). Authors NS and PRF are inventors on these applications.

\section{Authors' contributions}

NS and PRF conceived the study, analyzed and interpreted the data, and drafted the manuscript with the assistance of KB and YB. NS and YB generated plant material and performed physiological studies. $Y B$ and $K B$ performed gene expression analyses. KB assisted with microarray data analysis and with the preparation of figures and tables. All authors read and approved the final manuscript. 


\section{Acknowledgements}

We thank Kasi Williams for technical assistance, Larissa Ramsay and Chad Matsalla for bioinformatics support, Eddie Risseeuw and Raju Datla for pER330, Ken Nelson for PBI425, Irina Zaharia for hormone profiling, and Adrian Cutler and Fawzy Georges for critically reading the manuscript. This research was supported by funding from the National Research Council of Canada through the Genomics and Health Initiative. This publication is NRCC No. 54656.

Received: 23 November 2012 Accepted: 2 October 2013

Published: 29 October 2013

\section{References}

1. Boyer JS: Plant productivity and environment. Science 1982, 218:443-448.

2. Marris E: Water: more crop per drop. Nature 2008, 452:273-277.

3. Ludlow MM: Strategies of response to water stress. In Structural and functional responses to environmental stresses: water shortage. Edited by Kreeb KH, Richter H, Hinckley TM. The Hague, Netherlands: SPB Academic Publishing; 1989:269-281

4. Verslues PE, Agarwal M, Katiyar-Agarwal S, Zhu J, Zhu JK: Methods and concepts in quantifying resistance to drought, salt and freezing, abiotic stresses that affect plant water status. Plant J 2006, 45:523-539.

5. Drost DR, Benedict Cl, Berg A, Novaes E, Novaes CR, Yu Q, et al: Diversification in the genetic architecture of gene expression and transcriptional networks in organ differentiation of populus. Proc Natl Acad Sci U S A 2010, 107:8492-8497.

6. Zhang J, Nguyen HT, Blum A: Genetic analysis of osmotic adjustment in crop plants. J Exp Bot 1999, 50:291-302

7. Ingram J, Bartels D: The molecular basis of dehydration tolerance in plants. Annu Rev Plant Physiol Plant Mol Biol 1996, 47:377-403.

8. Tuteja N: Abscisic acid and abiotic stress signaling. Plant Signal Behav 2007, 2:135-138.

9. Yamaguchi-Shinozaki K, Shinozaki K: Organization of cis-acting regulatory elements in osmotic- and cold-stress-responsive promoters. Trends Plant Sci 2005, 10:88-94.

10. Yamaguchi-Shinozaki K, Shinozaki K: Transcriptional regulatory networks in cellular responses and tolerance to dehydration and cold stresses. Annu Rev Plant Biol 2006, 57:781-803.

11. Deyholos MK: Making the most of drought and salinity transcriptomics. Plant Cell Environ 2010, 33:648-654.

12. Huang D, Wu W, Abrams SR, Cutler AJ: The relationship of drought-related gene expression in arabidopsis thaliana to hormonal and environmental factors. J Exp Bot 2008, 59:2991-3007.

13. Moumeni A, Satoh K, Kondoh H, Asano T, Hosaka A, Venuprasad R, et al: Comparative analysis of root transcriptome profiles of two pairs of drought-tolerant and susceptible rice near-isogenic lines under different drought stress. BMC Plant Biol 2011, 11:174.

14. Harb A, Krishnan A, Ambavaram MM, Pereira A: Molecular and physiological analysis of drought stress in arabidopsis reveals early responses leading to acclimation in plant growth. Plant Physiol 2010, 154:1254-1271.

15. Seki M, Narusaka M, Ishida J, Nanjo T, Fujita M, Oono Y, et al: Monitoring the expression profiles of 7000 arabidopsis genes under drought, cold and high-salinity stresses using a full-length CDNA microarray. Plant $J$ 2002, 31:279-292.

16. Umezawa T, Okamoto M, Kushiro T, Nambara E, Oono Y, Seki M, et al: CYP707A3, a major ABA 8'-hydroxylase involved in dehydration and rehydration response in arabidopsis thaliana. Plant J 2006, 46:171-182.

17. Xie Z, Li D, Wang L, Sack FD, Grotewold E: Role of the stomatal development regulators FLP/MYB88 in abiotic stress responses. Plant J 2010, 64:731-739.

18. Hussain $\mathrm{S}$, lqbal M, Arif M, Amjad M: Beyond osmolytes and transcription factors: drought tolerance in via protective proteins and aquaporins. Biol Plantarum 2011, 55:401-413.

19. Saez A, Rodrigues A, Santiago J, Rubio S, Rodriguez PL: HAB1-SWI3B Interaction reveals a link between abscisic acid signaling and putative SWI/SNF chromatin-remodeling complexes in arabidopsis. Plant Cell 2008, 20:2972-2988

20. Qiao W, Fan L: Epigenetics, a mode for plants to respond to abiotic stresses. Front Biol 2011, 6:477-481.

21. Suzuki M, McCarty DR: Functional symmetry of the B3 network controlling seed development. Curr Opin Plant Biol 2008, 11:548-553.
22. Tsukagoshi H, Morikami A, Nakamura K: Two B3 domain transcriptional repressors prevent sugar-inducible expression of seed maturation genes in arabidopsis seedlings. Proc Natl Acad Sci U S A 2007, 104:2543-2547.

23. Tsukagoshi H, Saijo T, Shibata D, Morikami A, Nakamura K: Analysis of a sugar response mutant of arabidopsis identified a novel B3 domain protein that functions as an active transcriptional repressor. Plant Physiol 2005, 138:675-685.

24. Suzuki M, Wang HH, McCarty DR: Repression of the Leafy cotyledon 1/B3 regulatory network in plant embryo development by VP1/ABSCISIC Acid Insensitive 3-Like B3 genes. Plant Physiol 2007, 143:902-911.

25. Guerriero G, Martin N, Golovko A, Sundstrom JF, Rask L, Ezcurra I: The RY/Sph element mediates transcriptional repression of maturation genes from late maturation to early seedling growth. New Phytol 2009, 184:552-565.

26. Veerappan $\mathrm{V}$, Wang J, Kang M, Lee J, Tang Y, Jha AK, et al: A novel HSI2 mutation in arabidopsis affects the PHD-like domain and leads to derepression of seed-specific gene expression. Planta 2012, 236:1-17.

27. Rose PA, Cutler AJ, Irvine NM, Shaw AC, Squires TM, Loewen MK, et al: 8'-acetylene ABA: an irreversible inhibitor of ABA 8'-hydroxylase. Bioorg Med Chem Let 1997, 7:2543-2546.

28. Okamoto M, Kuwahara A, Seo M, Kushiro T, Asami T, Hirai N, et al: CYP707A1 and CYP707A2, which encode abscisic acid 8'-hydroxylases, are indispensable for proper control of seed dormancy and germination in arabidopsis. Plant Physiol 2006, 141:97-107.

29. Huang D, Jaradat MR, Wu W, Ambrose SJ, Ross AR, Abrams SR, et al: Structural analogs of $A B A$ reveal novel features of $A B A$ perception and signaling in arabidopsis. Plant J 2007, 50:414-428.

30. Cutler AJ, Rose PA, Squires TM, Loewen MK, Shaw AC, Quail JW, et al: Inhibitors of abscisic acid 8'Hydroxylase. Biochemistry 2000, 39:13614-13624.

31. Masle J, Gilmore SR, Farquhar GD: The Erecta gene regulates plant transpiration efficiency in arabidopsis. Nature 2005, 436:866-870

32. Yoo CY, Pence HE, Jin JB, Miura K, Gosney MJ, Hasegawa PM, et al: The arabidopsis GTL1 transcription factor regulates water use efficiency and drought tolerance by modulating stomatal density via transrepression of SDD1. Plant Cell 2010, 22:4128-4141.

33. Urano K, Maruyama K, Ogata Y, Morishita Y, Takeda M, Sakurai N, et al: Characterization of the ABA-regulated global responses to dehydration in arabidopsis by metabolomics. Plant $J$ 2009, 57:1065-1078.

34. Moulin M, Deleu C, Larher F, Bouchereau A: The lysine-ketoglutarate reductasesaccharopine dehydrogenase is involved in the osmo-induced synthesis of pipecolic acid in rapeseed leaf tissues. Plant Physiol Biochem 2006, 44:474-482.

35. Gouesbet G, Blanco C, Hamelin J, Bernard T: Osmotic adjustment in Brevibacterium ammoniagenes: pipecolic acid accumulation at elevated osmolalities. J Gen Microbiol 1992, 138:959-965.

36. Wilkins O, Brautigam K, Campbell MM: Time of day shapes arabidopsis drought transcriptomes. Plant J 2010, 63:715-727.

37. Cutler SR, Rodriguez PL, Finkelstein RR, Abrams SR: Abscisic acid: emergence of a core signaling network. Annu Rev Plant Biol 2010, 61:651-679.

38. Hong $Y$, Zheng $S$, Wang $X$ : Dual functions of phospholipase Dalpha1 in plant response to drought. Mol Plant 2008, 1:262-269.

39. Jiang H, Li H, Bu Q, Li C: The RHA2a-interacting proteins ANACO19 and ANAC055 may play a dual role in regulating ABA response and jasmonate response. Plant Signal Behav 2009, 4:464-466.

40. Wang Y, Ying J, Kuzma M, Chalifoux M, Sample A, McArthur C, et al: Molecular tailoring of farnesylation for plant drought tolerance and yield protection. Plant J 2005, 43:413-424.

41. Bergmann DC, Sack FD: Stomatal development. Annu Rev Plant Biol 2007, 58:163-181.

42. Yang Y, Costa A, Leonhardt N, Siegel RS, Schroeder JI: Isolation of a strong arabidopsis guard cell promoter and its potential as a research tool. Plant Methods 2008, 4:S1-S2.

43. Wang RS, Pandey S, Li S, Gookin TE, Zhao Z, Albert R, et al: Common and unique elements of the ABA-regulated transcriptome of arabidopsis guard cells. BMC Genomics 2011, 12:S1.

44. Zhang H, Bishop B, Ringenberg W, Muir WM, Ogas J: The CHD3 remodele PICKLE associates with genes enriched for trimethylation of histone $\mathrm{H3}$ lysine 27. Plant Physiol 2012, 159:418-432.

45. Lemaitre B, Nicolas E, Michaut L, Reichhart JM, Hoffmann JA: The dorsoventral regulatory gene cassette spatzle/toll/cactus controls the potent antifungal response in drosophila adults. Cell 1996, 86:973-983.

46. Kemphues K: Essential genes. WormBook (The C elegans Research Community, WormBook) 2005:1-7. http://www.wormbook.org. 
47. Kagale S, Rozwadowski K: EAR motif-mediated transcriptional repression in plants: an underlying mechanism for epigenetic regulation of gene expression. Epigenetics 2011, 6:141-146.

48. Alonso JM, Stepanova AN, Leisse TJ, Kim CJ, Chen H, Shinn P, et al: Genomewide insertional mutagenesis of arabidopsis thaliana. Science 2003, 301:653-657.

49. Karimi M, Inze D, Depicker A: GATEWAY vectors for agrobacteriummediated plant transformation. Trends Plant Sci 2002, 7:193-195.

50. Clough SJ, Bent AF: Floral dip: a simplified method for agrobacteriummediated transformation of arabidopsis thaliana. Plant J 1998 $16: 735-743$

51. Chiwocha SD, Abrams SR, Ambrose SJ, Cutler AJ, Loewen M, Ross AR, et al: A method for profiling classes of plant hormones and their metabolites using liquid chromatography-electrospray ionization tandem mass spectrometry: an analysis of hormone regulation of thermodormancy of lettuce (Lactuca sativa L.) seeds. Plant J 2003, 35:405-417.

52. Oliver MJ, Guo L, Alexander DC, Ryals JA, Wone BW, Cushman JC: A sister group contrast using untargeted global metabolomic analysis delineates the biochemical regulation underlying desiccation tolerance in sporobolus stapfianus. Plant Cell 2011, 23:1231-1248.

53. Smyth GK: Limma: linear models for microarray data. In Bioinformatics and computational biology solutions using $R$ and bioconductor. Edited by Gentleman R, Carey V, Dudoit S, Irizarry R, Huber W. New York: Springer; 2005:397-420.

54. Sharma N, Anderson M, Kumar A, Zhang Y, Giblin EM, Abrams S, et al: Transgenic increases in seed oil content are associated with the differential expression of novel brassica-specific transcripts. BMC Genomics 2008, 9:619.

doi:10.1186/1471-2229-13-170

Cite this article as: Sharma et al:: High-level expression of sugar inducible gene2 (HSI2) is a negative regulator of drought stress tolerance in Arabidopsis. BMC Plant Biology 2013 13:170.

\section{Submit your next manuscript to BioMed Central and take full advantage of:}

- Convenient online submission

- Thorough peer review

- No space constraints or color figure charges

- Immediate publication on acceptance

- Inclusion in PubMed, CAS, Scopus and Google Scholar

- Research which is freely available for redistribution 